\title{
FINE STRUCTURE OF PHOTOSPHERIC FACULAE
}

\author{
R. MULLER \\ Pic Du Midi Observatory \\ 65200 Bagnères de Bigorre
}

\begin{abstract}
Properties of the photospheric bright points associated with magnetic flux tubes are reviewed both in faculae (facular points) and in the photospheric network (network bright points - NBPs) out of active regions. A special attention is given to their size distribution, to their location relative to the granular, mesogranular and supergranular patterns, and to their relation with the small scale magnetic features, both in active and quiet regions. In particular a new granulation movie reveals that NBPs form in large intergranular spaces, compressed by the surrounding granules.

At the center of the solar disk, bright points are much brighter than the mean photosphere; their contrast increases toward the $\operatorname{limb}$ up to $\mu=0.3-0.2$ and then decreases to the limb, as it is now widely accepted. But, all the published contrasts are of little significance because of center-to-limb selection effects. New center-to-limb contrast variations of individual network bright points are presented, which take into account the selection effects.
\end{abstract}

\section{Introduction}

According to their historical definition, faculae are extended bright areas visible in active regions when they are close enough to the solar limb. High resolution observations show that faculae are formed with many small features, most of them being smaller than 1 " (Figure 1, from Muller, 1975), called facular points or facular granules (Bray and Loughead, 1964, Muller, 1975, 1977). Facular points are difficult to study near the limb because of foreshortening. It isn't very easy to identify facular features in white light near the disk center because their contrast does not exceed the contrast of granules. Nevertheless, in the core of photospheric lines or in the wings of strong chromospheric lines like $\mathrm{H} \alpha$ or CaIIH and $\mathrm{K}$, facular features can be observed very well at the disk center, as their contrast increases with the height in the photosphere. In addition these sub-arcsecond features are not affected by the foreshortening when they are far away from the limb. Actually, instead of using narrow filters in Fraunhofer lines, I use a quite wide $10 \mathrm{~A}$ bandpass interferential filter centered on the $\mathrm{CN}$ band at $4308 \mathrm{~A}$; it allows me to observe with exposure times which are short enough to resolve successfully subarcsecond features down to the diffraction limit of the telescope $\left(\mathrm{O}^{\prime \prime} .2\right.$ : Figures 2 and 4$)$. Facular points are produced by magnetic flux tubes. Consequently they can be used to locate the magnetic field in the photosphere, which is very difficult on magnetograms because of insufficient resolution; this allows us to obtain indirect important information about the interaction between flux tubes and convective patterns at granular, mesogranular and supergranular scales, and to derive the physical structure of flux tubes from their contrast.

Bright photospheric features are not only observed in active regions but also in the quiet atmosphere of the sun, where they form the photospheric network, which is cospatial with supergranule boundaries. Faculae are also visible at the solar poles, especially around minima of activity when the polar magnetic field is relatively strong; they are known as polar faculae. 


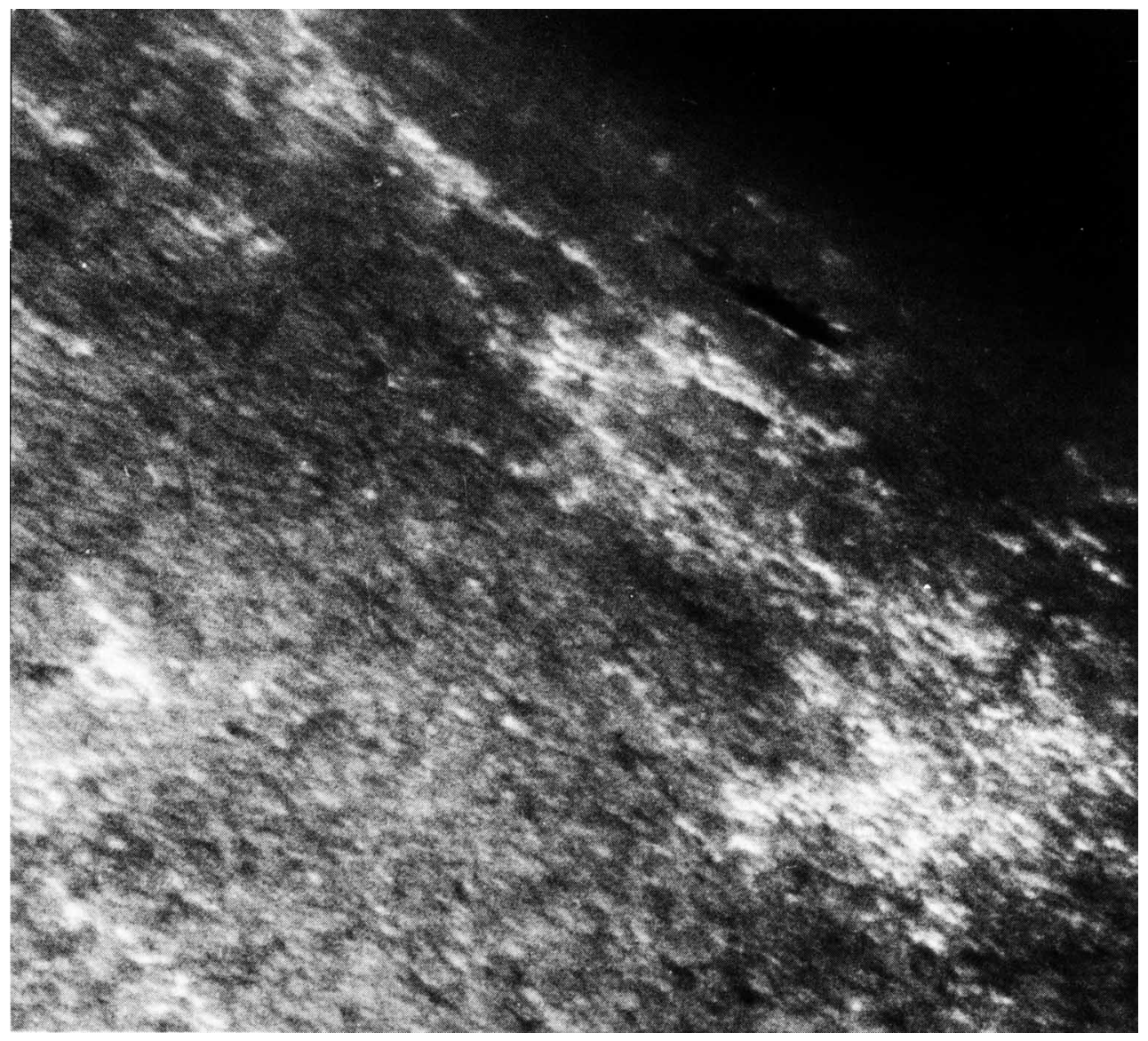

Figure 1. White light faculae near the solar limb.

Both the faculae in active regions and the photospheric network in the quiet sun are formed of bright points, most of them being smaller than O".5. In quiet and active regions, their properties (size, brightness, lifetime) are very similar and they are believed to have the same magnetic origin (Chapman and Sheeley, 1968). In the photosphere, network bright points often appear isolated or surrounded by only a few neighbours. In active regions the situation is more complicated because the density of facular points is very high, forming a compact pattern of adjacent points.

Consequently the basic properties of facular points will be much easier to study in the quiet sun ; they will be described in the next section. Then the properties of facular points in active regions will be compared to those of network bright points.

The facular contrast and its center-to-limb variation deserves a particular attention because they are used to derive empirical facular and magnetic flux tube models, as well as to test theoretical models. It will be shown that the center-to-limb variations published so far are of little significance and cannot be used to derive models; more significant and realistic new measurements will be presented. 


\section{Properties Of Network Bright Points (NBPs) In The Quiet Photosphere}

At the photospheric level, the size of NBPs rarely exceeds $\mathrm{O}^{\prime \prime} .5$ (Figure 2) ; their characteristic size has been found to be close to O"2 - $150 \mathrm{~km}-$ (Muller and Keil, 1983; Mehltretter, 1974 ; Dunn and Zirker, 1973), it may even be smaller if the size of many NBPs is beyond the resolution of existing telescopes. They are located in the intergranular lanes (Mehlhetter, 1974 ; Muller, 1983, Figure 2) and at the supergranular boundaries (Muller, 1983) ; no clear relation with the mesogranular pattern is known, except that they avoid diverging regions in the granule horizontal proper motions (Figure 3).

A detailed description of the behavior of NBPs has been given by Muller (1983) : they appear at the supergranular boundaries, seldom inside the cells ; on a smaller scale they are formed in large spaces at the junction of several granules, never inside a granule nor in a common space between two granules : they appear preferentially at the boundaries of convective cells. The lifetime ranges from 5 to $50 \mathrm{~min}$, with an average value of 18 min; their size rarely exceeds $\mathrm{O}^{\prime .} .5$; they remain in intergranular spaces throughout their lifetime ; they tend to form very close to an already existing bright point ; $15 \%$ of them seem to split into two facular points; they disappear simply by fading away in an intergranular space ; merging with another bright point has not been clearly observed. It is also worth mentioning that granules surrounding NBPs are disturbed during their formation phase : they become elongated in the direction of the NBP, pointing to it (Muller, Roudier, Hulot, 1989).

\section{Properties of facular Points (FPs) In Active Regions}

\subsection{MORPHOLOGY}

In active regions, faculae extend over large areas and are mainly formed of chains and of compact clusters of tiny bright points, when observed at very high resolution like in Figure 4 (which was taken in the $\mathrm{CN}$ band at $4308 \mathrm{~A}$ ); the basic feature appears to be a very small bright point smaller than $\mathrm{O}^{\prime .5}$, even than $\mathrm{O}^{\prime \prime .3}$ for many of them. These facular points (FPs) are very similar to the NBPs described above, in size, brightness and lifetime (Muller and Mena, 1986). They can be aligned forming chains, crinkles (Dunn and Zirker, 1973) and clusters of adjacent points a few arcseconds in size. Their relationship with granules (which are significantly smaller than in the quiet sun) is not very clear, although many of them are located in intergranular lanes. In the continuum, the granulation appears smeared if the seeing is not perfect (abnormal granulation, Dunn and Zirker, 1973). Facular points form a pattern of cells, 2" to 5 " in size, including several granules (Title, Tarbell, Topka, 1987) ; why such a cellular pattern shows up is still unknown. Supergranular size cells can also be delineated. Unlike in the quiet sun, the size of FPs can significantly exceed $\mathrm{O}^{\prime \prime} .5$ or even 1 "O in a few cases (Figure 4). According to Spruit and Zwaan (1981) and Schüssler and Solanki (1983), facular points of size in the range $0 " .5-1.5$ should not be bright; this point should be clarified. Near the limb probably only large and bright FPs and unresolved clusters of small FPs are visible (see section 5).

\subsection{RELATION TO THE PHOTOSPHERIC MAGNETIC FIELD}

At moderate resolution, down to about 1", both the photospheric network and faculae coincide well with the photospheric magnetic field (Chapman and Sheeley, 1968). At higher resolution the detailed correspondence is less clear; there is however strong 


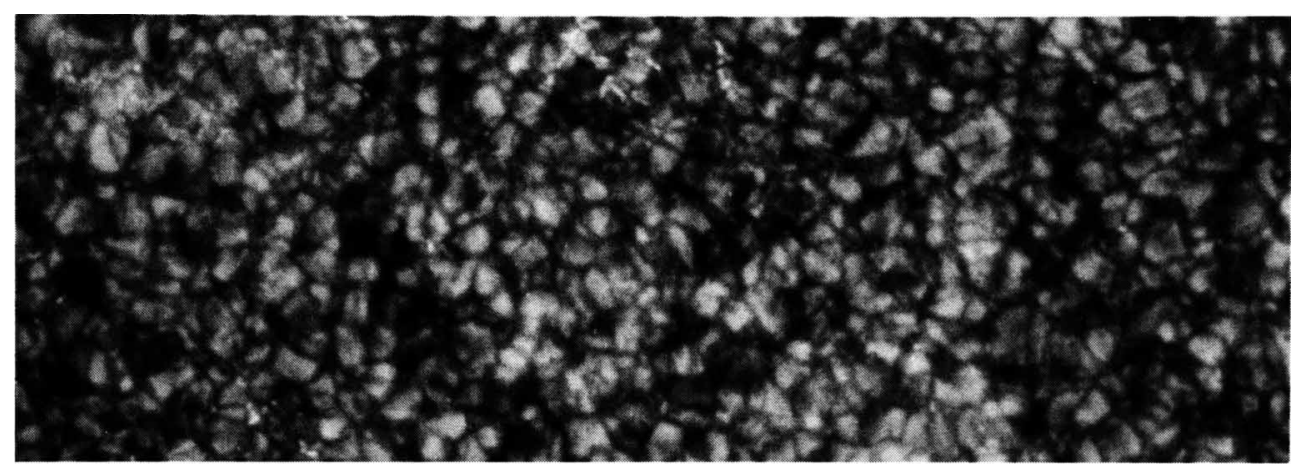

Figure 2. Network Bright Points (NBPs) imbedded in the granular pattern. Filtergram taken at $4308 \AA$ in the $C N$ band.

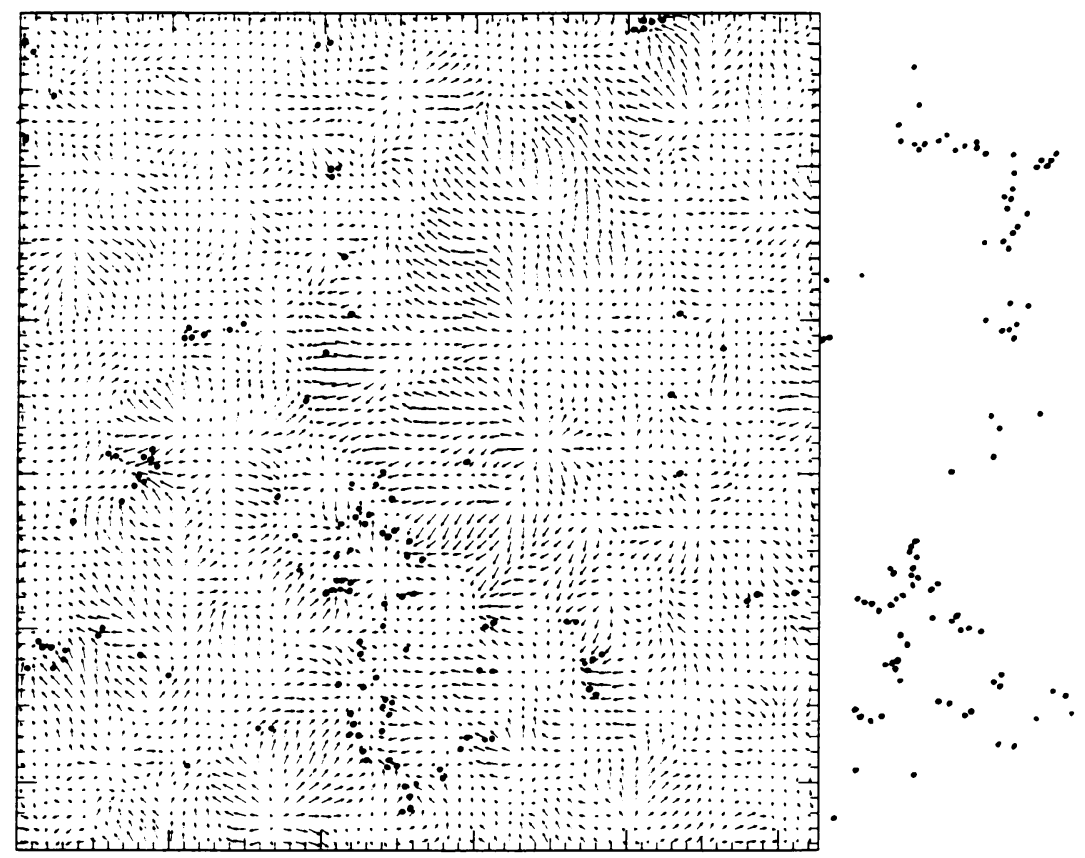

Figure 3. Location of NBPs in the granular flow pattern in a quiet area (55" x 53") at the disk center. The flow pattern was derived from an outstanding granulation movie obtained at the Pic du Midi Observatory and processed with the Title technique at Lokheed Palo Alto Research Laboratory (Title et al. 1989). NBPs concentrate at the boundary of a large supergranule which is also delineated by large outward flows ; they also concentrate in several converging flow regions. The large concentration of NBPs in the lower half of the area corresponds to an abnormal granulation area. Many diverging flow regions can be recognized easily in the processed area ; they could correspond to mesogranules; NBPs seem to avoid these diverging regions. There are very few NBPs identified inside the large supergranule. 


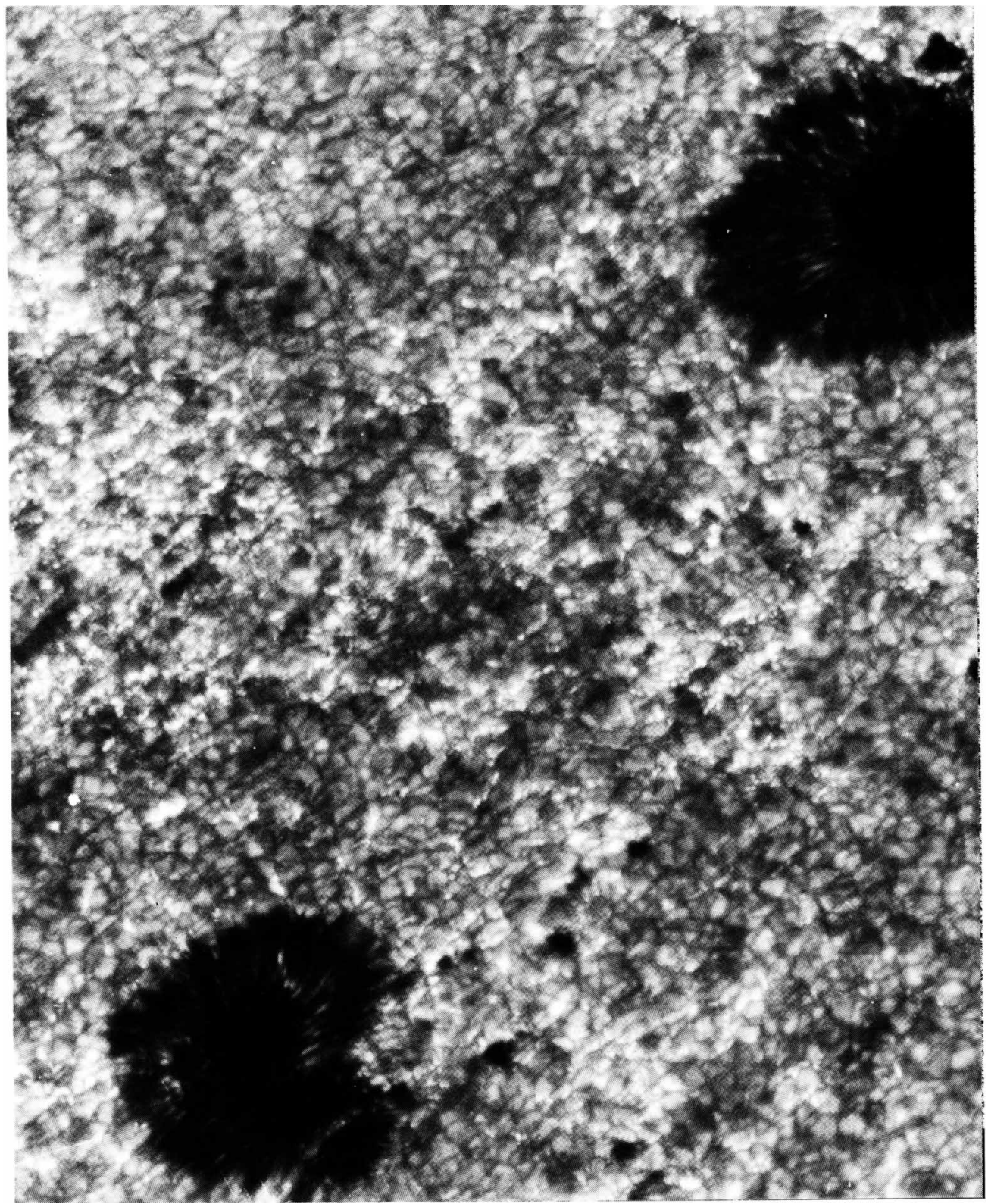

Figure 4. Photospheric faculae observed near the disk center, with the $50 \mathrm{~cm}$ refractor at the Pic du Midi Observatory, in the $\mathrm{CN}$ band at 4308 A (10 A bandbass filter). Most facular points are smaller than $0 " .5$, many of them approaching the diffraction limit of the telescope $(0 " .2)$; on the other hand larger bright features, sometimes exceeding $1 " .0$ are not rare. 
evidence that NBPs and FPs are closely related to kG magnetic fields (Stenflo, 1973, Title et AL.1985). The close relation is confirmed by new high resolution magnetograms obtained by Title and co-workers at La Palma (private communication); however the magnetic field cross-section seems to be a little larger than that of the corresponding bright points. In fact, a close inspection of Figure 3 shows that adjacent bright points are very close to each other ; this means that if every bright point is associated to a flux tube, its cross-section cannot be much larger than the size of the point.

In the quiet sun, very few NBPs can be identified inside supergranule cells (Muller, 1983) Figure 3 in this paper. Nevertheless intranetwork magnetic field can be detected with sensitive magnetographs (Harvey, 1977, Martin, 1988). Why ? The magnetic flux associated with an NBP is $4 \times 10^{17} \mathrm{Mx}$ if we assume a field strength of $2000 \mathrm{G}$ and a diameter of $150 \mathrm{~km}$. The magnetic flux measured in the intranetwork field is about $10^{16}$ Mx, which means that either the size or the field strength is much smaller than for the magnetic features visible at the supergranule boundaries. This may explain why flux tubes are not visible in filtergrams as bright points : either their size is too small to be resolved with the best $50 \mathrm{~cm}$ class solar telescopes, or the magnetic field is too weak for a significant brightening to be produced.

\section{Contrast At The Disk Center}

Faculae are visible as bright features in white light only near the limb. Near the center of the disk, their observed brightness is about the same as that of granules, which makes them uneasy to identify. Muller and Keil (1983) have measured an average brightness of 1.08 times the mean brightness of the photosphere. However, owing to their very small size, their real brightness should be much higher ; actually, Muller and Keil (1983) derived an average value corrected for blurring, in the range $1.3-1.5$; it can even be much higher for the brightest NBPs. It seems that the background intensity of the photosphere in facular areas is slightly lower than in the quiet sun (Hirayama, Hamano and Mizugaki, 1985).

In photospheric lines or in the wings of chromospheric lines, faculae are visible as bright features even at the disk center.

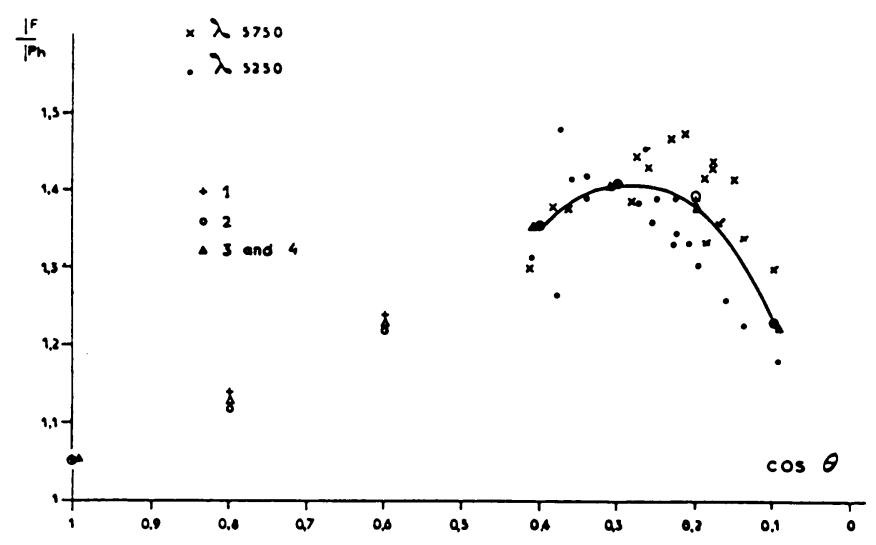

Figure 5. Center-to-Limb Variation of the intensity of facular points (relative to the sunsunding quiet photosphere), corrected for blurring.

Observation : Pic du Midi $50 \mathrm{~cm}$ refrator; resolution : 0".3 (Muller, 1975, Figure 3). 


\section{Center-To-Limb Variation of The Contrast}

The center-to-limb variation of the contrast is used to derive empirical facular and flux tube models or to test theoretical models. This explains why it is an important parameter to be measured accurately.

Most of the published Center-to-Limb Variations (CLV) of the contrast of faculae were obtained from low resolution measurements. That kind of variations are useful for solar oblateness determinations and for evaluating the contribution of faculae to the energy budget of active regions (variation of solar irradiance and luminosity problem). The only high resolution CLV published so far is the one by Muller (1975), who claimed a resolution of $\mathrm{O}^{\prime \prime} .3$. He found a maximum of contrast of 1.4 (corrected for blurring) near $\mu=0.3$, then a decrease toward the limb (Figure 5). The extrapolated value at the disk center is strongly underestimated, owing to the measurements of Muller and Keil (1983). The Muller's CLV is confirmed by Hirayama (1978) who analysed O".8 resolution observations.

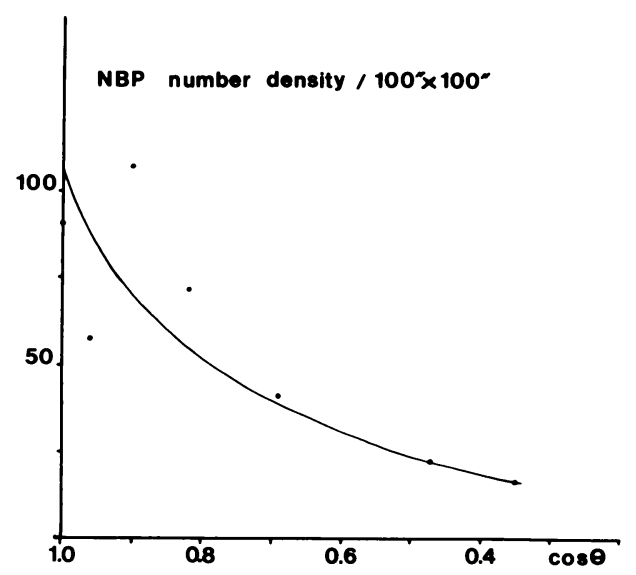

Figure 6. Center-to-Limb Variation of the number density of NBPs number per surface unit of $\left.100^{\prime \prime} \times 100 "\right)$.

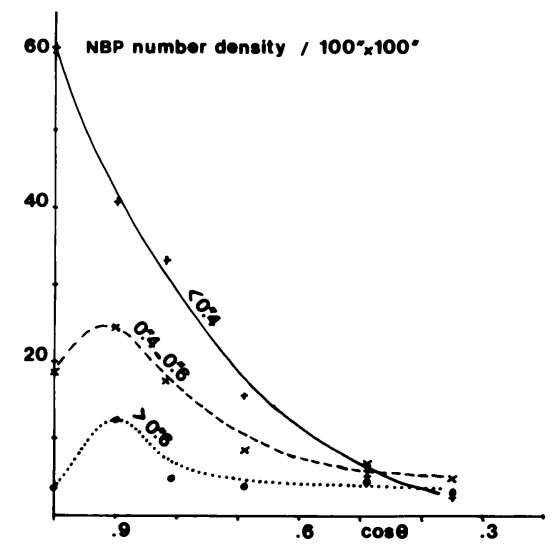

Figure 7. Center-to-Limb Variation fo the number of NBPs of various classes of sizes. 
CLV of facular contrast have to be used with caution when deriving models, for the following reason pointed out by Muller and Roudier (1984): the number of NBPs decreases between the center of the disk and the limb by a factor as large as 90 or $95 \%$ (Figure 6 ; Figure 5a in Muller and Roudier, 1984). This means that most NBPs are not visible at the limb, because of the combined effect of foreshortening and resolution, or because of transparency if faculae are elevated hot clouds as suggested by cloud models (Hirayama and Moriyama, 1979 ; Stenflo and Solanki, 1980). This also means that near the limb the visible facular features are the largest ones (selection effect), or are clusters of adjacent unresolved NBPs, especially in active regions where all published measurements were made. Thus even Muller's high resolution CLV measurements do not correspond to isolated characteristic NBPs and are then also irrelevant for deriving and testing facular and flux tube models.

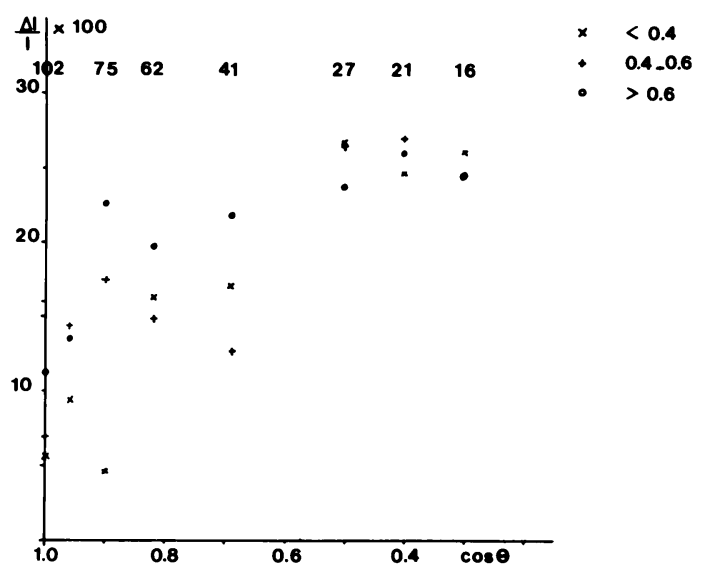

Figure 8. Center-to-Limb Variation of the contrast, all NBPs included. The number density of identified NBPs at various positions on the disk is indicated below the curve.

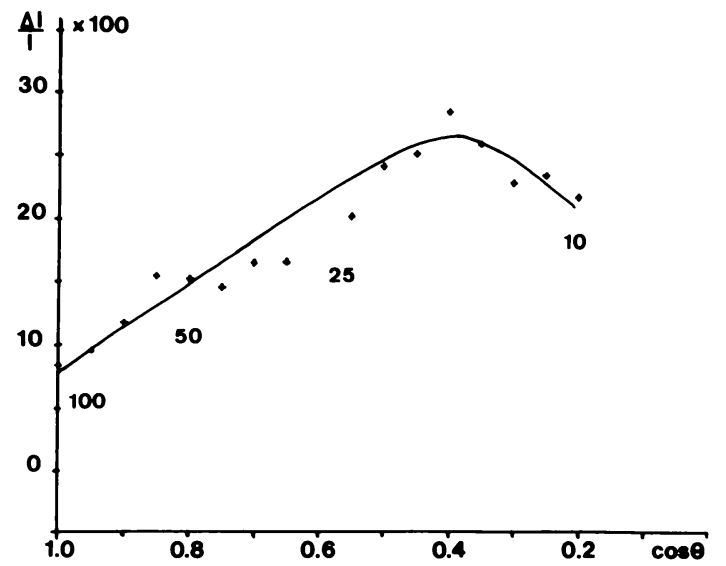

Figure 9. Center-to-Limb Variation of the contrast, for NBPs of various classes of size. The number density of identified at various positions on the disk is indicated above the curve. 


\section{Center-to-Limb Variation of Isolated NPBs}

Figure 7 shows the varation of the number of NBPs of different sizes : as expected NBPs smaller than $0 " .4$ desappear much faster than the larger ones. The increase of the number of points larger than $0 " .4$ around $\mu=0.9$ is still unexplained. Near the center of the disk, NBPs smaller than $0 " .4$ are dominant; near the limb there are less. The variation of the average brightness (i.e. all NBPs, large, small, bright and weak included, Figure 8) is very similar to that $I$ obtained for facular elements in active regions. A part of the contrast increase is due to the selection effect mentionned above : at the center of the disk about 100 NBPs per surface unit of $100 "$ " $100 "$ are identified; as we move toward the $\operatorname{limb}$, this number decreases to a value of about 10 . The variation is almost size independant (Figure 9), although near the disk center large NBPs seem to be brighter in average than the small ones; there is almost no brightness difference between large and small NBPs near the limb; however the selection is still effective, especially for the points smaller than $0 " .4$ (see Figure 7). Therefore it is hard to know the CLV of a typical bright point of a given size, except for the brightest ones. Because near the limb only 5 NBPs are identified in each class of size, we may plot the contrast variation of the five brightest NBPs present at each observed heliographic position (Figure 10). Again the CLV is almost size independant (however the CLV for each class of size is very unaccurate because of the low number of bright NBPs visible near the limb). The contrast increase from the center of the disk to $\mu=0.3$ is relatively flatter than for the case when all NBPs are included (Figure 8), because of the higher brightness at the disk center. Toward the limb the contrast decreases. Very probably the contrast should also increase rather smoothly for weaker NBPs. Models should take this smooth increase into account, resulting probably in a smaller vertical temperature gradient inside flux tubes.

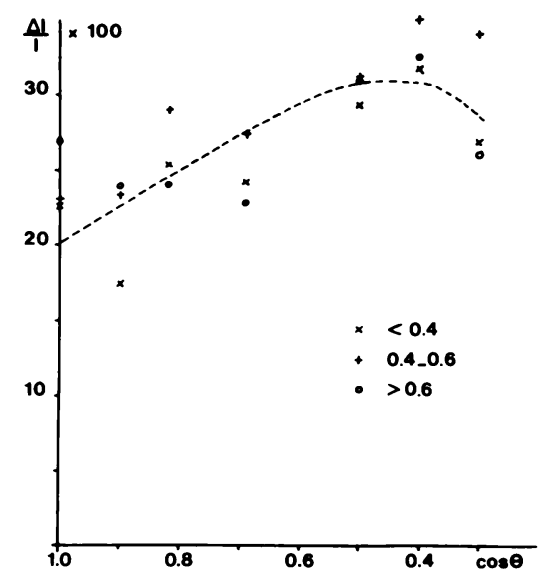

Figure 10. Center-to-Limb Variation of the contrast of the five brightest NBPs of each class of size and at every observed position on the solar disk.

\section{Addendum : formation of NBPs by granule compression}

The movie from which we have derived the granulation flow pattern shown in Figure 3 has also been used to study the dynamics of the formation of NBPs. As no simultaneous CaIIK3933 or CN4308 filtergrams have been performed, NBPs have been directly 


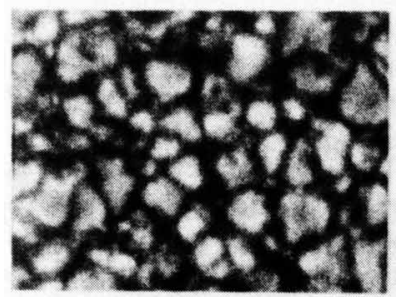

0

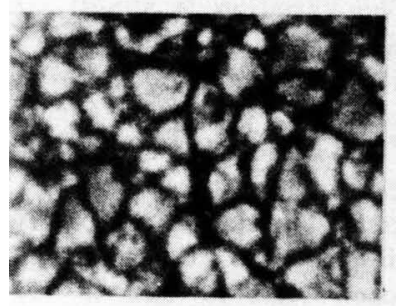

$\mathrm{t}=1 \mathrm{~min}$

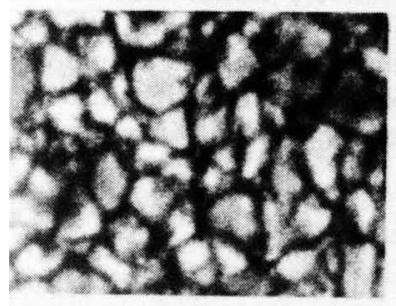

2

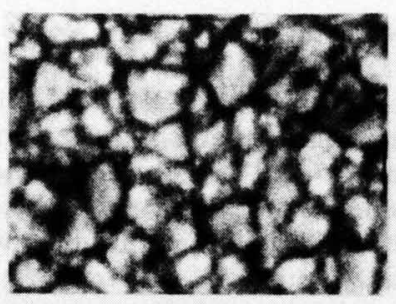

3

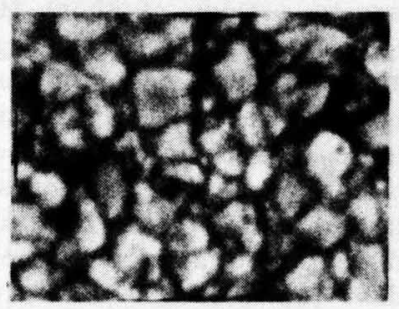

4

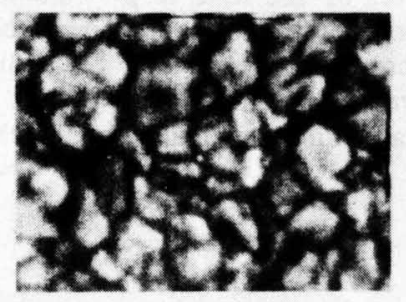

5

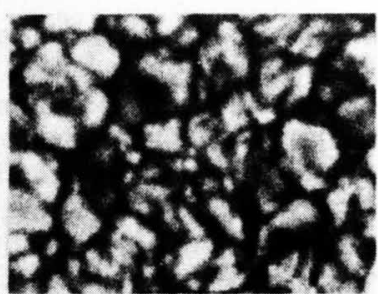

6

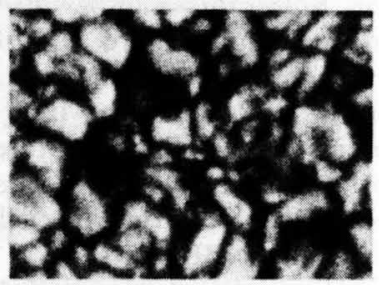

7

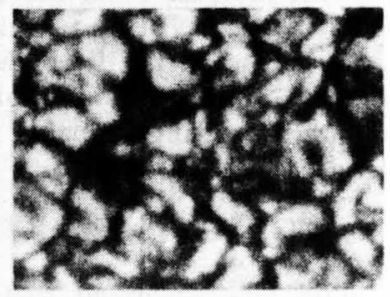

8

Figure 11. Formation of an NBP in the center of a large intergranular space by the compression of the converging surrounding granules (in the center of the field of view). Its maximum of visibility accurs after 5 minutes.

identified from the white light movie as small (smaller than $0 " .5$ ), relatively bright and sharp features, of lifetimes longer than $10 \mathrm{~min}$ (small granules with which they can be mistaken have much shorter lifetimes, less than $3 \mathrm{~min}$ ). NBPs appear in large intergranular spaces at the junction of several granular when they converge towards the center of the space (Figure 11) ; their brightness increases during the process and reaches a maximum when they are compressed by the surrounding granules, which form at this stage a "daisy-like pattern" (Figure 11, at $\mathrm{t}=1,2,3,4,5 \mathrm{~min}$; several conspicuous examples are also shown in Figure 2 in the paper by Muller, Roudier and Hulot, 1989). When the surrounding granules evolve, the daisy-like pattern is destroyed; the NBPs remain visible in the intergranular lanes for another $15 \mathrm{~min}$, on an average, although they are less bright and less sharp; they undergo a chaotic random walk controlled by evolving granules, especially the expanding ones. In general, when a large space appears in the intergranular pattern, for example when a large granule fades away, the surrounding granules quickly fill this "empty" space ; usually nothing special occurs, but if some magnetic flux is present there (problably not concentrated enough to be visible as an 
NBP), it can be compressed and concentrated by the converging granules, producing an NBP. Thus, NBPs probably correspond to a concentration phase of the magnetic flux. If most of the magnetic field in the photosphere has a strength of 1 to $2 \mathrm{kG}$, as inferred from the line ratio technique (Stenflo,1973), then the strength may even reach higher values during the concentration phase ; another possibility which cannot be excluded is that of a normally rather weak magnetic field, being concentrated to kilogauss strengths only for a while during the phase of granule compression. In order to understand the formation of flux tubes properly, it will be necessary to mesure the evolution of the magnetic field and the flow during the granule compression process with a spatial resolution well below $0 " .5$.

\section{Conclusion}

The importance of the photospheric faculae lies in the fact that they are produced by magnetic fields. They allow us to learn about the structure and the behavior of the magnetic flux tubes. However some basic properties like the size, the brightness, the relation to the magnetic field, are not yet known with sufficient accuracy and detail ; the center-to-limb variation of the contrast of a typical NBP or FP is not even known at all. The spatial resolution of the observations have to be improved for filtergrams as well as for magnetograms ; this requires : a) telescopes larger than the $50 \mathrm{~cm}$ ones currently in operation ; b) shorter exposure times which can now be obtained with CCD cameras ; c) image stabilization devices or telescopes in the space. From a theoretical point of view, faculae and flux tube models should be revised so that they produce a flatter center-to$\operatorname{limb}$ variation of the contrast between $\mu=1.0$ and $\mu=0.3$.

Acknowledgements.

H. Auffret has participated in the analysis of the center-to-limb variation of the contrast of NBPs. The granulation movie has been digitized and processed at Lockheed Palo Alto Research Laboratory in collaboration with Th. Roudier, J. Vigneau, Z. Frank, R. Shine, T. Tarbell and A. Title and at the Solar National Observatory at Sunspot in collaboration with G. Simon. They are all aknowledged for their kind hospitality in the United States. A financial support was obtained from the US Air Force, through a Window-On-Science trip WOS-89-0047.

\section{References}

Bray, R.J. and Loughhead, R.E. (1964), Sunspots, Chapman and Hall, Ltd, London.

Chapman, G.A. and Sheeley, N.R. (1968) 'The photospheric network', Solar Phys. 5, 442-

Dunn, R.B. and Zirker, J.B. (1973) 'The solar filigree', Solar Phys. 33, 281-304.

Harvey, J.W. (1977) 'Photospheric magnetic and velocity fields in active regions', in E.A. Müller (ed.), Highlights of Astronomy, 4, 223 .

Hirayama, T. (1978) Publ. Astron. Soc. Japan 30,337.

Hirayama, T., Hamano, S. and Mizugaki, K. (1985) 'Precise wideband photometry of photospheric faculae with an emphases on the disk center', Solar Phys. 99, 43. 
Hirayama, T. and Moriyama, F. (1979) 'Center-to-limb variation of the intensity of the photospheric faculae', Solar Phys. 63, 251.

Martin, S.F. (1988) 'The identification and interaction of network, intranetwork and ephemeral-region magnetic fields', Solar Phys. 117, 243-259.

Mehltretter, J.P. (1974) 'Observations of photospheric faculae at the center of the solar disk', Solar Phys. 38, 43-57.

Muller, R. (1975) 'A model of photospheric faculae deduced from white light high resolution pictures', Solar Phys. 45, 105-114.

Muller, R. (1977) 'Morphological properties and origin of the photospheric facular granules', Solar Phys. 52, 249-262.

Muller, R. (1983) 'The dynamical behavior of facular points in the quiet photosphere', Solar Phys. 85, 113-121.

Muller, R. and Keil, S.L. (1983) 'The characteristic size and brightness of facular points in the quiet photosphere', Solar Phys. 87, 243-250.

Muller, R. and Ména B. (1987) 'Motions around a decaying sunspot', Solar Phys. 112, 295-303.

Muller, R. and Roudier, Th. (1984) 'Variability of the quiet photospheric network', Solar Phys. 94, 33-47.

Muller, R., Roudier, Th. and Hulot, J.C. (1989) 'Perturbation of the granular pattern by the presence of magnetic flux tubes', Solar Phys. 119, 229-243.

Schüssler, M. and Solanki, S.K. (1988) 'Continuum intensity of magnetic flux concentrations : are magnetic elements bright points?', Astron. Astrophys. 192, 338-342.

Spruit, H.C. and Zwaan, C. (1981) 'The size dependance of contrasts and numbers of small magnetic flux tubes in an active region', Solar Phys. 70, 207-228.

Stenflo, J.O. (1973) 'Magnetic-field structure of the photospheric network', Solar Phys. 32, 41-63.

Title, A.M., Tarbell, T.O. and Topka, K.P. (1987) 'On the relation between magnetic field structures and granulation', Astrophys. J. 317, 892-899. 